
Journal of Education, Health and Sport. 2020;10(5):213-217. eISSN 2391-8306. DOI http://dx.doi.org/10.12775/JEHS.2020.10.05.022 https://apcz.umk.pl/czasopisma/index.php/JEHS/article/view/JEHS.2020.10.05.022 https://zenodo.org/record/3870920

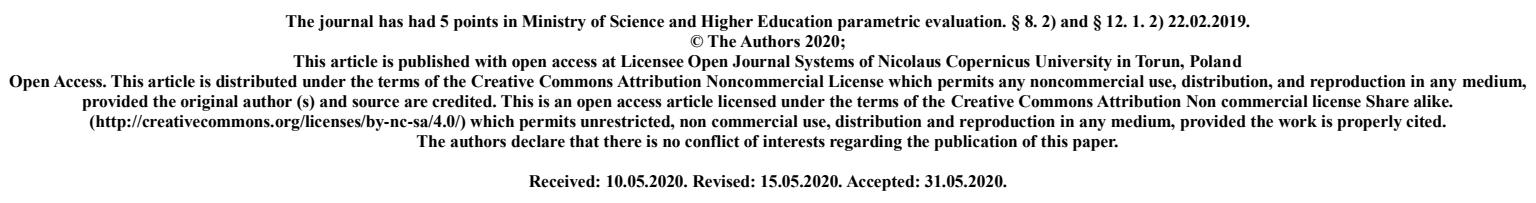

\title{
Infectious complications of peritoneal dialysis
}

\author{
Paweł Polski ${ }^{1}$, Monika Kusz ${ }^{2}$, Paulina Pawluczuk², Agnieszka Maślak², Adam Alzubedi ${ }^{1}$ \\ 1. Department of General and Transplant Surgery and Nutritional Treatment, Medical \\ University of Lublin
}

2. Department of Pediatric Nephrology, Medical University of Lublin

\section{Address fot correspondence:}

Paweł Polski, Department of General and Transplant Surgery and Nutritional Treatment, Medical University of Lublin, 8 Jaczewskiego St, 20-954 Lublin, Poland, phone: +48 514746 457, e-mail: maestro532@wp.pl

\begin{abstract}
:
Peritoneal dialysis is one of three basic methods of renal replacement therapy. Others include hemodialysis and kidney transplantation. In peritoneal dialysis, the peritoneal cavity is used to clean the blood of excess unnecessary substances and water. The peritoneum is a thin membrane permeable to water and many substances, lining the organs inside the abdominal cavity. Briefly, the peritoneum can be considered a kind of filter. When performing peritoneal dialysis, a dialysis fluid is administered to the abdominal cavity, which discharges after some time, and this activity is repeated at planned intervals. The chemical composition of the fluid is appropriately selected for each patient so as to obtain optimally favorable results of dialysis. Water diffusion depends on the concentration in the fluid of glucose and other osmotic factors. Among the infectious complications of peritoneal dialysis, including drainage site infections, tunnel inflammation and peritonitis associated with peritoneal dialysis, the latter remains the most common and serious.
\end{abstract}

Key words: peritoneal dialysis, peritonitis, tenckhoff catheter, infectious complications 


\section{Introduction:}

Among the infectious complications of peritoneal dialysis, there are catheter site inflammation, tunnel inflammation and peritonitis. Severe and prolonged episodes of peritonitis lead to a structural remodeling of the peritoneal membrane, thereby leading to its failure. Peritonitis occurs with a frequency of up to $30 \%$ and is the main reason for this method's failure (1). Many patients rightly treat peritonitis as a life-threatening situation requiring hospitalization. The most common symptoms of the disease include severe, diffuse abdominal pain, fever, weakness and cloudy dialysis fluid recovered from the peritoneum (2). The latest ISPD (International Society of Peritoneal Dialysis) guidelines stress the need for regular, annual monitoring of the incidence of peritonitis. In addition, it is recommended to monitor the frequency of individual microorganisms and their antibiotic sensitivity. Depending on the antibiotic sensitivity, the empirical antibiotic therapy regimen may also be modified (3).

\section{Procedure:}

The principle of peritoneal dialysis is to administer to the peritoneal cavity through a previously implanted Tenckhoff catheter fresh dialysis fluid and to release it after a specified period of time. The fluid change is painless for the patient. After filling the peritoneal cavity, the catheter is closed. During this period, the patient can function without significant restrictions. After a few hours, a new empty plastic bag is connected to the catheter into which the used fluid flows. After dropping it, fresh liquid is again given. This procedure for manual fluid exchange is called Continuous Ambulatory Peritoneal Dialysis (CADO). Usually, the patient alone or with the help of a family conducts 3-4 such fluid exchanges during the day and leaves the peritoneal cavity filled with fluid for the night. A special device - a cycler - can also be used to change the fluid. This technique is called automatic peritoneal dialysis (APD). Numerous exchanges using the cycler usually take place at night while the patient is asleep. During the day, the peritoneal cavity is left without dialysis fluid or you can do 1-2 manual dialysis. The cycler is a small device that the patient can easily take with him on a journey. The decision on the choice of technique is made by the doctor together with the patient and his family. The number of exchanges is closely matched to each patient and can be modified. The patient is constantly in contact with the center conducting the course of his treatment. Patients who want to travel are not strictly limited by peritoneal dialysis. However, at the destination they should have adequate conditions for replacements as well as professional medical assistance in the event of complications.

\section{Diagnosis:}

Dialysis peritonitis should be suspected in any patient reporting cloudy dialysate fluid. The criteria for its recognition include 2 components from the following 3:

1. Clinical manifestations of peritonitis: abdominal pain, cloudy dialysis fluid.

2. Pleocytosis (the number of white blood cells exceeding 100 in 1 ul dialysate, including over $50 \%$ of multinucleated blood cells).

3. Positive culture of dialysate

Ready-made kits with suitable media can be used to collect the microbiological swab. It is important that in centers where the number of sterile peritonitis exceeds $15 \%$, a thorough 
analysis of the method of collecting microbiological material should be made (3). After securing the material, empirical antibiotic therapy, usually consisting of two antibiotics, should be started as soon as possible. One targeted for Gram-positive bacteria (1st generation cephalosporin, vancomycin), the other for the presence of Gram-negative bacteria (3rd generation cephalosporin or aminoglycoside). The most commonly recognized pathogens in a given center are of great importance in the selection of antibiotics. Antibiotics can be administered both intravenously and intraperitoneally (4). Intraperitoneal supply is the recommended route. In contrast, the intravenous route is used only for cases of generalized sepsis. The length of treatment used while maintaining stable drug doses is also important. It turns out that after the administration of an intraperitoneal dose of vancomycin, in the absence of peritonitis, about $50 \%$ of the dose is absorbed into the bloodstream, and about $90 \%$ during inflammation. It is recommended that the interval between doses be 4-5 days (5). Although the monitoring of vancomycin serum levels is somewhat controversial, it is recommended that it be given and at concentrations below $15 \mu \mathrm{g} / \mathrm{ml}$, administration immediately.

An important issue is the choice of peritoneal dialysis technique during ongoing peritonitis. Many authors recommend that you convert the technique used by the patient to the CADO method. It is also a fact that we now have less data on the dosage and demand for antibiotics in the case of dying automatic methods. However, it should be remembered that with the supply of antibiotics during one exchange per day (exchange during the day), using the cycler overnight is associated with a greater peritoneal clearance of antibiotics than in the case of CADO. It often happens that with a temporary change of the technique to CADO, the patient becomes overhydrated, which may lead to the need to stop this technique. In one retrospective observational study, there were no differences in the frequency of relapses, mortality, or the need to remove the dialysis catheter between patients treated with APD and CADO and continuing their treatment regimen. However, in the group treated automatically, the higher cytosis of the dialysis fluid and the duration of treatment of the inflammatory episode lasted longer (6).

The percentage of elderly people undergoing peritoneal dialysis is currently increasing. In this group, this type of renal replacement therapy is beneficial because it is less haemodynamically demanding and well tolerated. Data from numerous publications do not show that old age is a significant factor in the increase in the incidence of dialysis peritonitis. In contrast, the incidence of drainage site infections and catheter tunnel inflammation is significantly higher. During the treatment of peritonitis, side effects of antibiotic therapy are more often observed, especially in the neurotoxic effects of third generation cephalosporins. Older people may be more likely to require peritoneal dialysis during this time, and an episode of inflammation may affect mortality. It was also found that a large number of comorbidities are associated with a higher frequency of peritonitis recurrences in this group (7). 


\section{Prevention:}

1. Training programs

Much space in the latest ISPD guidelines is devoted to the issue of peritoneal training, which is often a key element of a properly functioning peritoneal dialysis program. It is recommended that such training be carried out by qualified nursing staff in accordance with the developed protocol (8). It was established that the place of training can be the patient's home, clinical hospital or any other hospital unit prepared for conducting the training and equipped with appropriate equipment and materials. The training organization is flexible, but the course should not be interrupted for more than 2 days. Training can take place either before or after the Tenckhoff catheter is implanted. Regardless of the patient's first training at the start of renal replacement therapy, its repetition plays a huge role in reducing the frequency of infectious complications. (9).

\section{Tenckhoff catheter implantation}

One of the two most important recommendations is the preventive use of antibiotic therapy prior to implantation of a peritoneal dialysis catheter. Cefazolin is most commonly used in clinical practice. Perioperative systemic, prophylactic antibiotic administration has been shown to reduce the incidence of peritonitis in the early post-catheter implantation period (10). There were no differences in the frequency of peritonitis depending on the method of catheter implantation (laparoscopic method vs. standard laparotomy) or type of catheter (1112).

3. Care for the catheter outlet

Currently, persistent topical antibiotic therapy at the Tenckhoff catheter outlet is not used. Each patient is properly trained on the antiseptic of the catheter site. In addition, with any disturbing symptom (redness, inflammatory infiltration, soreness) the patient should report to the leading center. (13)

4. Secondary prevention

Prevention of the development of fungal peritonitis is important here, because this complication, although rare, is associated with significant mortality, reaching even $20-30 \%$. In $40 \%$ of other patients with this infection irreversible changes in the peritoneal membrane occur, preventing the continuation of this method of renal replacement therapy. Candida albicans is the most common isolated pathogen, and standard treatment regimens, apart from immediate removal of the Tenckhoff catheter, include fluconazole, amphotericin B and flucytosine, alone or in combination, depending on the sensitivity of the pathogen (14).

\section{Summary:}

Peritoneal dialysis is a good solution for renal replacement therapy for patients who want to be professionally active as well as for the elderly. It provides a smaller hemodynamic load for the body. However, like all methods, it carries the risk of certain complications. Post-dialysis peritonitis is one of the most serious complications. Updated in 2016 by ISPD, guidelines for the prevention and management of dialysis peritonitis significantly improved the prognosis for peritonitis. They pay particular attention to the patient's training system, care of the outlet, perioperative systemic administration of the antibiotic during the dialysis catheter implantation procedure and secondary antifungal prophylaxis in the case of bacterial peritonitis. Each dialysis center, after analyzing data on the frequency of infectious 
complications, the pathogens causing them, and their sensitivity, should adopt guidelines according to their local conditions.

\section{References:}

1. ANZDATA Registry. 38th Report. Chapter 5: Peritoneal Dialysis. Australia and New Zealand Dialysis and Transplant Registry, Adelaide, Australia 2016.

2. Campbell D.J., Craig J.C., Mudge D.W. i wsp. Patients' perspectives on the prevention and treatment of peritonitis in peritoneal dialysis: a semi-structured interview study. Perit. Dial. Int. 2016; 36: 631-639.

3. Li P.K., Szeto C.C., Piraino B. i wsp. ISPD Peritonitis Recommendations: 2016 Update on Prevention and Treatment. Perit. Dial. Int. 2016; 36: 481-508.

4. Flanigan M.J., Lim V.S. Initial treatment of dialysis associated peritonitis: a controlled trial of vancomycin versus cefazolin. Perit. Dial. Int. 1991; 11: 31-37.

5. Fish R., Nipah R., Jones C., Finney H., Fan S.L. Intraperitoneal vancomycin concentrations during peritoneal dialysis-associated peritonitis: correlation with serum levels. Perit. Dial. Int. 2012; 32: 332-338.

6. Rüger W., van Ittersum F.J., Comazzetto L.F., Hoeks S..E, ter Wee P.M. Similar peritonitis outcome in CAPD and APD patients with dialysis modality continuation during peritonitis. Perit. Dial. Int. 2011; 31: 39-47.

7. Szeto C.C. Peritoneal dialysis-related infection in the older population. Perit. Dial. Int. 2015; 35: 659-662.

8. Figueiredo A.E., Bernardini J., Bowes E. i wsp. A syllabus for teaching peritoneal dialysis to patients and caregivers. Perit. Dial. Int. 2016; 36: 592-605.

9. Dong J., Chen Y. Impact of the bag exchange procedure on risk of peritonitis. Perit. Dial. Int. 2010; 30: 440-447.

10. Strippoli G.F., Tong A., Johnson D., Schena F.P., Craig J.C. Antimicrobial agents to prevent peritonitis in peritoneal dialysis: a systematic review of randomized controlled trials. Am. J. Kidney Dis. 2004; 44: 591-603.

11. Hagen S.M., Lafranca J.A., Ijzermans J.N., Dor F.J. A systematic review and metaanalysis of the influence of peritoneal dialysis catheter type on complication rate and catheter survival. Kidney Int. 2014; 85: 920-932.

12. Tullavardhana T., Akranurakkul P., Ungkitphaiboon W., Songtish D. Surgical versus percutaneous techniques for peritoneal dialysis catheter placement: a meta-analysis of the outcomes. Ann. Med. Surg. (Lond.) 2016; 10: 11-18.

13. Figueiredo A.E., de Mattos C., Saraiva C. i wsp. Comparison between types of dressing following catheter insertion and early exit-site infection in peritoneal dialysis. J. Clin. Nurs. 2017; doi: 10.1111/jocn.13738.

14. Matuszkiewicz-Rowinska J. Update on fungal peritonitis and its treatment. Perit. Dial. Int. 2009; 29 (supl. 2): S161-S165. 